\title{
Significación pronóstica y utilidad clínica de la determinación sérica e inmunohistoquímica de catepsina B en cáncer colorrectal
}

\author{
D. PADILLA, T. CUBO, J. M. MOLINA, M. GARCÍA ${ }^{1}$, G. DE LA OSA ${ }^{2}$ \\ T. PALOMINO ${ }^{2}$, R. PARDO, J. MARTÍN, E. ARÉVALO ${ }^{3}$, J. HERNÁNDEZ CALVO
}

Servicio de Cirugía General y del Aparato Digestivo. Complejo Hospitalario de Ciudad Real. ${ }^{1}$ Servicio de Anatomía Patológica. ${ }^{2}$ Servicio de Análisis Clínicos. ${ }^{3}$ Servicio de Cirugía General y Aparato Digestivo. Hospital Reina Sofía. Cordoba

\begin{abstract}
PROGNOSTIC SIGNIFICANCE AND CLINIC UTILITY OF SERUM AND INMUNOHISTOCHEMICAL CATHEPSIN B LEVELS IN COLORECTAL CANCER
\end{abstract}

\section{RESUMEN}

Introducción: Aproximadamente un tercio de los enfermos con cáncer colorrectal sin metastatización ganglionar recidivan debido a la existencia de micrometástasis no detectadas con los métodos histológicos habituales. La expresión de ciertas enzimas implicadas en el proceso metastásico del cáncer colorrectal como la catepsina B, puede predecir el potencial metastásico y mala evolución de las neoplasias en etapas tempranas para considerar terapias más agresivas. Nuestro objetivo es establecer el valor pronóstico de la determinación sérica e inmunohistoquímica de catepsina $\mathrm{B}$, con el fin de identificar los carcinomas colorrectales más agresivos

Métodos: Estudio transversal y cohorte de 55 enfermos con cáncer colorrectal intervenidos quirúrgicamente en nuestro servicio de forma electiva entre 1998 y 2000. Utilizamos como grupo control, 23 enfermos intervenidos quirúrgicamente por apendicitis aguda. Se realizó determinación sérica preoperatoria de catepsina B (KRKA,dd Novo Slovenia; ng/ml) e inmunohistoquímica tras resección quirúrgica, (C-19, Santa Cruz Biotechnology). El resto de marcadores utilizados fueron CEA, (INMULIT 2000 CEA;5 ng/ml); CA 19,9, (INMULITE GI-M;37 UI/ml); p53, (Dako ).El análisis de supervivencia se realizó mediante los métodos de Cox y KaplanMeier, con el paquete estadístico SPSS 10.0 para Windows.

Resultados: La edad media de los enfermos con cáncer colorrectal fue 68 años, 39-87. 29 fueron varones y 26 mujeres. El tamaño de los tumores fue 4,6 cm, 1-12. La localización más frecuente fue rectal, 32,2\%, y la diferenciación histológica moderada, $49,1 \%$. Los valores séricos e inmunohistoquímicos de los enfermos con apendicitis aguda fueron $5,74 \mathrm{ng} / \mathrm{ml} \mathrm{y}$ $29,56 \%$ respectivamente. Los valores séricos preoperatorios en enfermos con cáncer colorrectal fueron: CEA; 46,04 ng/ml (0,21-7,32); CA 19,9; 110,52UI/ml, (2,5-1920), y catepsina B: $6,94 \mathrm{ng} / \mathrm{ml}(3,57-11,6)$

Los valores inmunohistoquímicos fueron p53: 44,36\% (0-95); catepsina B tisular, 66,9\%, (10-90). Los valores séricos e inmunohistoquímicos de catepsina B fueron significativamente superiores en enfermos con cáncer colorrectal respecto al grupo control, $\mathrm{p}=0,011 \mathrm{y} \mathrm{p}=0,000$ respectivamente. Los valores preoperatorios séricos de catepsina $\mathrm{B}$ se relacionaron significativamente con una menor supervivencia del enfermo tanto en modelo univariante como multivariante, $\mathrm{p}=0,041 ; \mathrm{HR} 1,281,95 \%$ IC $(1,010-1,624) \mathrm{y}$ $\mathrm{p}=0,022 ;$ HR $1,338,95 \%$ IC $(1,043-1,716)$.

Conclusiones: La catepsina B puede ser considerado como marcador pronóstico independiente en cáncer colorrectal. Su determinación sérica preoperatoria, con valores por encima de $6,94 \mathrm{ng} / \mathrm{ml}$, se relaciona con una menor supervivencia del enfermo.

PALABRAS CLAVE: Catepsina B. Cáncer colorrectal. Marcador pronóstico.
ABSTRACT

Background: Aproximately one third of node-negative colorectal cancer recur suggesting the presence of micrometastasis not detected by conventional histopathologic methods. We think that the role of enzymes like Cathepsin B play in the process of invasion and metastasis in colorectal cancer might identify at earlier stages patients with high risk of shorter survival and who need more aggressive treatment. Our porpuse is to evaluate the prognostic significance of preoperative serum and inmunohistochemical levels of cathepsin B to identify colorectal carcinomas with worse prognostic.

Methods: Fifty five patients undergoing surgical treatment for colorectal cancer from 1998 to 2000. As a control group sera from 23 patients with acute appendicitis. Serum levels of cathepsin B were obtained preoperatively (KRKA, Novo Slovenia;ng/ml); cathepsin B inmunoreactivity was determinated after surgical treatment, (C-19, Santa Cruz Biotechnology). Serum levels of CEA (Inmulit 2000 CEA), and CA 19,9 (Inmulite Gi-Ma, Diagnostic Products Corporation, Los Angeles, CA), cancer. Survival analysis was realized using Cox and Kaplan-Meier methods (SPSS 10.0 for Windows).

Results: The mean age of patients with colorectal cancer was 68 years (range 39-87 years). 29 males and 26 females. Tumor size was 4.6 cms., range 1-12. Rectal localization, 32.2\%. Moderately differentiated, 49.1\%. The median serum and inmunohistochemical levels of cathepsin $B$ were $5.74 \mathrm{ng} / \mathrm{ml}$ and $29.56 \%$ in patients with acute appendicitis respectively. Preoperative serum levels in patients with colorectal cancer were: CEA, $46.04 \mathrm{ng} / \mathrm{ml}$ (range 0.21-7.32 ); CA 19,9, $110.52 \mathrm{UI} / \mathrm{ml}$, (range 2.5-1920); and Cathepsin B, $6.94 \mathrm{ng} / \mathrm{ml}$, range 3.57-11.6). Inmunohistochemical results were: p53, 44.36\%, (range 0-95); Cathepsin B, $66.9 \%$ (range 10-90). Serum and inmunhistochemical values were significantly increased in patients with colorectal cancer when compared with control group, $p=0,011$ and $p=0,000$. High serum levels of cathepsib $B$ were significantly associated wiyh shorter survival of patients with colorectal cancer in univariate and multivariate methods, $p=0.041 ; H R$ $1.28195 \%$ CI (1.043-1.716) and $p=0.022$; HR 1.338.955 CI (1.0431.716).

Conclusions: Cathepsin B can be used like an independent prognostic tumoral marker in colorectal cancer. Preoperative serum levels over $6.94 \mathrm{ng} / \mathrm{ml}$, are associated with worse prognostic and shorter survival.

KEY WORDS: Cathepsin B. Colorectal cancer. Prognostic marker. and 533 expression (Dako) were determinated in patients with colorectal

Padilla D, Cubo T, Molina JM, García M, De la Osa G, Palomino T, Pardo R, Martín J, Arévalo E, Hernández Calvo J. Significación pronóstica y utilidad clínica de la determinación sérica e inmunohistoquímica de catepsina B en cáncer colorrectal. An Med Interna (Madrid) 2003; 20: $521-525$.

Trabajo aceptado: 26 de junio de 2003

Correspondencia: David Padilla Valverde. Urb. Los Girasoles, 16-1 ${ }^{\text {a }}$ Fase. 13005 Ciudad Real. e-mail: maynona@terra .es 


\section{INTRODUCCIÓN}

El cáncer colorrectal representa el $13 \%$ respecto al resto de enfermedades neoplásicas malignas, siendo el responsable de hasta un $10 \%$ de los fallecimientos por cáncer.La supervivencia global a los cinco años del cáncer colorrectal es del $50 \%$, sin embargo estos datos se verán radicalmente modificados dependiendo de la extensión de la enfermedad maligna. La supervivencia será del 90\%, si sólo existe afectación local, disminuyendo considerablemente hasta 58 y $5 \%$ si existe metastatización regional y a distancia respectivamente (1). La existencia de micrometástasis de células neoplásicas colorrectales en un estadio precoz de la enfermedad y supuestamente de buen pronóstico, permite que la evolución del enfermo sea mala incluso una vez realizado tratamiento quirúrgico con intención curativa. Hasta un $30 \%$ de enfermos con cáncer colorrectal sin metastatización ganglionar fallecen por recidiva de la enfermedad. Son enfermos que en el caso de presentar localización cólica, no se benefician de tratamiento adyuvante.

La detección de estas neoplasias colorrectales de gran agresividad haría posible una terapia más agresiva con intención curativa.Con este motivo hemos intentado conocer el valor pronóstico de la catepsina B y su utilidad clínica, con el fin de identificar aquellos enfermos con mayor riesgo que puedan beneficiarse de terapias más agresivas o nuevas terapias, genética y/o inmunológica.

\section{PACIENTES Y MÉTODOS}

Entre 1998 y 2000 realizamos un estudio transversal y cohorte de un total de 78 enfermos, 23 enfermos con diagnóstico de apendicitis aguda y 55 enfermos con diagnóstico de cáncer colorrectal, intervenidos quirúrgicamente en nuestro Servicio, para determinar el valor pronóstico de la determinación preoperatoria sérica e inmunohistoquímica de Catepsina $\mathrm{B}$, respecto a marcadores séricos utilizados en nuestra actividad clínica diaria como son CEA, antígeno carcinoembrionario, y CA 19,9, y el marcador inmunohistoquímico p53.

Las variables estudiadas en los enfermos intervenidos quirúrgicamente con diagnóstico de apendicitis fueron, edad, sexo, catepsina B plasmática, catepsina B histológica y estadificación anatomopatológica.Las variables estudiadas en los enfermos intervenidos quirúrgicamente con diagnóstico de cáncer colorrectal fueron, edad, sexo, tratamiento quirúrgico, tamaño tumoral, localización tumoral, diferenciación neoplásica, estadificación histológica, metastatización ganglionar, CEA,antígeno carcinoembrionario, preoperatorio, CA19,9 preoperatorio, catepsina B preoperatoria, p53, catepsina B tisular, recidiva local y/o a distancia de la enfermedad neoplásica colorrectal y mortalidad.

Metodología de determinación de CEA.-La determinación preoperatoria de valores de CEA sérico, se realizó mediante la utilización del analizador automático INMULITE 2000, mediante el ensayo INMULIT 2000 CEA (Diagnostic Products Corporation, Los Angeles, CA), que representa un enzimoinmunoensayo quimioluminiscente, secuencial en dos etapas, de amplificación tipo "sándwich", heterogéneo, cuya fase sólida se compone de "beads" de anticuerpo policlonal antiCEA unido a fosfatasa alcalina. El sustrato quimioluminiscente es, nuevamente, un ester de fosfato de adamantil dioxoeta- no. Unidades definidas: $\mathrm{ng} / \mathrm{ml}$. El valor de corte habitual de 5 $\mathrm{ng} / \mathrm{ml}$ abarca el percentil $95,05 \%$ de los individuos sanos.

Metodología de determinación de CA19,9.-La determinación preoperatoria de CA19,9 se realizó mediante el analizador automático INMULITE con el ensayo INMULITE GI-MA (Diagnostic Products Corporation, Los Angeles, $\mathrm{CA}$ ), que representa un enzimoinmunoensayo quimioluminiscente de dos pasos, con procedimiento de amplificación tipo "sándwich", heterogéneo, cuya fase sólida está constituida por " beads” de poliestireno recubiertas con anticuerpo monoclonal específico para CA 19,9, (anticuerpo de captura) y que utiliza como anticuerpo marcado, anticuerpo monoclonal murino anti-CA 19,9, unido covalentemente a fosfatasa alcalina y como sustrato un ester fosfato de adamantil dioxoetano. Unidades definidas: $\mathrm{U} / \mathrm{ml}$. El valor de corte de $37 \mathrm{u} / \mathrm{ml}$ representa el percentil 99,4\% de individuos sanos.

Metodología de determinación de p53.-Una vez resecada la pieza tumoral y realizadas las técnicas histológicas e inmunohistoquímicas protocolizadas, las muestras se incubaron con el anticuerpo anti-p53 (clon D07, Dako, prediluido), durante una hora a temperatura ambiente, prosiguiendo normalmente con el método Envision.

Metodología de determinación plasmática de catepsina B.-La h-catepsina B se determinó mediante un enzimoinmunoensayo secuencial en dos pasos, de amplificación, tipo "sándwich", heterogéneo, con fase sólida tipo microplaca con un anticuerpo de captura policlonal IgG antih-cathepsina $\mathrm{B}$ procedente de conejo, utilizando como anticuerpo marcado anti h-catepsina procedente de oveja, unido a peroxidasa de rábano (KRKA, dd Novo mesto Slovenia). Las placas se leyeron en un lector Boehlisa (Ingelheim Diagnóstica y Tecnología S.A., Barcelona).Unidades definidas: $\mathrm{ng} / \mathrm{ml}$.

Metodología de determinación inmunohistoquímica de catepsina B.-Las secciones para el estudio inmunohistoquímico de $4 \mathrm{~m}$ de grosor fueron desparafinadas mediante técnica habitual y sometidas a desenmascaramiento antigénico mediante microondas durante 15 minutos. Se diseñó un protocolo específico mediante el sistema Envision, que incluyó los pasos habituales, como la inactivación de preoxida endógena, y la exposición al anticuerpo primario de cabra anticatepsina B humana (C-19, Santa Cruz Biotechnology), a una dilución 1.3000, durante 60 minutos. Como anticuerpo puente se utilizó IgG de conejo anti-cabra a una dilución 1:500 (Jackson InmunoResearch Laboratoires,Inc.), durante 15 minutos.Posteriormente se realizó la técnica Envision (Dako) automatizada idéntica a la utilizada con otros anticuerpos policlonales.

Se realizaron secciones de control negativo si no se observaba inmunotinción o esta se detectaba en menos de un $1 \%$ de las células. Existió expresión baja de catepsina B, cuando menos del $25 \%$ de las células tumorales eran positivas.La expresión se consideró alta, con inmunorreacción en más del 25\% de las células neoplásicas (Fig. 1). En nuestro estudio se realizó una cuantificación de la expresión positiva.Las células epiteliales y estromales se evaluaron por separado.

Plan de trabajo.-Seleccionamos para nuestro estudio 55 enfermos con diagnóstico preoperatorio de cáncer colorrectal, 55 enfermos,intervenidos quirúrgicamente de forma electiva en nuestro Servicio. Todos los enfermos fueron sometidos a un estudio completo endoscópico del colon y recto en régimen 


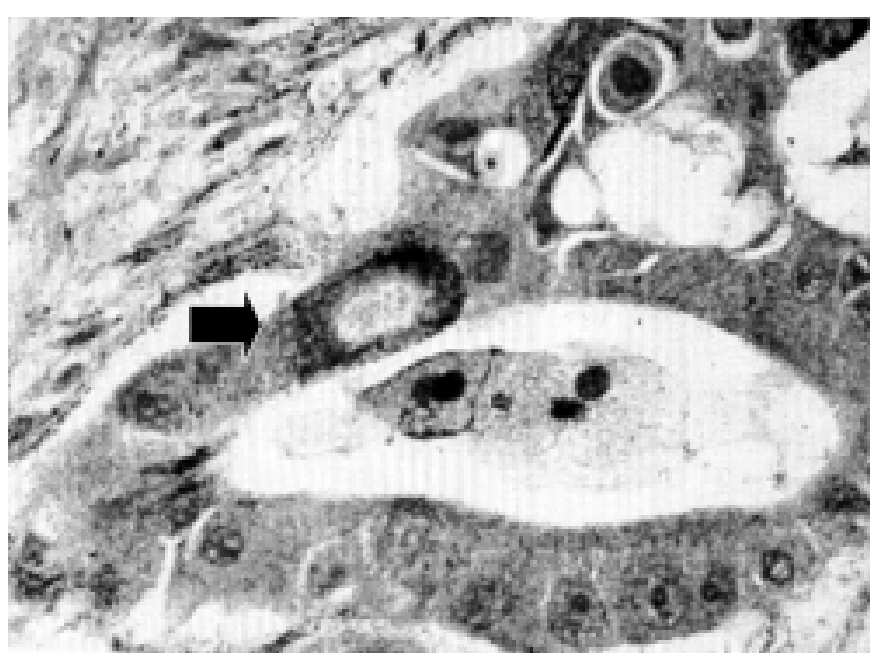

Fig. 1. Adenocarcinoma moderadamente diferenciado de intestino grueso. Fuerte tinción citoplasmática difusa de células neoplásicas epiteliales de disposición glandular y cordonal. Observese reacción algo más débil en las células estromales. Catepsina B x200.

ambulatorio tras preparación habitual y completa colorrectal. Se realizó biopsia incisional preoperatoria en todas las ocasiones. Todos los enfermos fueron ingresados al menos 24 horas antes de la intervención quirúrgica con idea de realizar preparación mecánica colorrectal preoperatoria, realizar profilaxis tromboembólica, mediante el uso de heparina de bajo peso molecular, y profilaxis antibiótica. Del mismo modo estos enfermos fueron informados de la naturaleza de la enfermedad y riesgos asociados a la realización de un tratamiento quirúrgico, y certificaron su consentimiento para ser incluidos en este estudio.

La extracción de muestras sanguíneas se realizó 24 horas antes de la intervención quirúrgica. La determinación de los valores de CEA, CA 19,9 y catepsina B se realizó según los métodos definidos, por el Servicio de Análisis Clínicos del Complejo Hospitalario de Ciudad Real.

Una vez resecada la pieza tumoral se realizaron las técnicas histológicas e inmunohistoquímicas para la determinación de los valores de p53 y catepsina B tisular según las técnicas previamente descritas, por el Servicio de Anatomía Patológica del Complejo Hospitalario de Ciudad Real.

Se realizó el tallado de las piezas quirúrgicas, seleccionando material, procurando que incluyese tejido neoplásico y borde con epitelio intestinal no neoplásico, así como suficiente pared intestinal en profundidad. Este material una vez fijado en formol tamponado al $10 \%$, se procesó de forma habitual en bloques de parafina. Se realizaron cortes de $4 \mathrm{~m}$ de grosor con tinción mediante hematoxilina-eosina.El estudio inmunohistoquímico se realizó mediante un equipo automático polivalente (Envision, Dako Corp.), previo desenmascaramiento antigénico. Como cromógeno se usó DAB (Dako). Se utilizó el sistema de clasificación histológica de Astler y Coller modificado.La valoración en todos los enfermos fue realizada por dos patólogos que deconocían la historia clínica del enfermo en cada caso.La utilización de tratamiento adyuvante fue llevado a cabo según protocolos de actuación del Servicio de Oncología del Complejo Hospitalario de Ciudad Real. Los enfermos una vez dados de alta hospitalaria se incluyeron en un programa de seguimiento con carácter ambulatoria y flexible ante la características del enfermo.

Análisis estadístico.Se utilizó la prueba de T de Student para muestras independientes, previa utilización del test de Kormogorov Smirnoff, para conocer la relación existente entre los valores plasmáticos preoperatorios e inmunohistoquímicos de catepsina $\mathrm{B}$ en enfermos con cáncer colorrectal respecto a patología colónica benigna,enfermos con diagnóstico de apendicitis.El análisis de los valores de catepsina B plasmáticos preoperatorios e inmunohistoquímicos respecto a los diferentes estadios del cáncer colorrectal se realizó mediante el análisis de varianza,ANOVA. El análisis de recidiva y mortalidad del enfermo se realizó mediate los metódos de Cox y Kaplan-Meier,log-rank test.Todas las pruebas estadísticas se realizaron con un nivel de significación estadístico del 95\%.Todos los cálculos estadísticos se realizaron a través del paquete estadístico SPSS 10,0 para Windows.

\section{RESULTADOS}

De los 55 enfermos intervenidos quirúrgicamente con diagnóstico de cáncer colorrectal, entre 1998,con un tiempo de seguimiento medio de 31 meses, 4-44, 29 fueron varones y 26 mujeres, con edad media de 68 años, 39-87. El tamaño medio de los tumores fue 4,6 cm 1-12. La localicación de la neoplasia colorretal fue cecal $(n=1)(1,8 \%)$, colon ascendente $(n=12) \quad(21,8 \%)$, colon transverso $(n=2) \quad(3,6 \%)$, colon decendente $(n=12)(21,8 \%)$, sigma $(n=7) \quad(12,7 \%)$, recto $(\mathrm{n}=21)(38,2 \%)$. Las neoplasias presentaron buena diferenciación en 25 ocasiones $(45,5 \%)$, moderada diferenciación $(n=27)(49,1 \%)$ y mala diferenciación $(n=3)(5,5 \%)$. La estadificación de las neoplasias fue la siguiente, A,2; B1,7; B2,22; C1,2; C2,16; D,6. En 16 ocasiones, 29,1\%, existió recidiva de la enfermedad y 18 enfermos fallecieron, $32,7 \%$. El valor sérico e inmunohistoquímico medio del grupo control fue 5,74 $\mathrm{ng} / \mathrm{ml}$ y $29,56 \%$ respectivamente. Los valores séricos preoperatorios fueron: CEA,46,04 $\mathrm{ng} / \mathrm{ml}, \quad(0,21-732)$; CA19,9, 110,52 U/ml, (2,5-1920); catepsina B,6,94 ng/ml, (3,57-11,6). Los valores inmunohistoquímicos fueron: p53, 44,36\%, (095); catepsina B tisular, 66,9\%, (10-90).

Los valores de catepsina B plasmáticos en enfermos con cáncer colorrectal fueron significativamente superiores a los hallados en enfermos con apendicitis aguda $(p=0,011)$.

Los valores inmunohistoquímicos de catepsina $\mathrm{B}$ en enfermos con cáncer colorrectal fueron significativamente superiores a los hallados en enfermos con apendicitis aguda $(\mathrm{p}=0,000)$.

Los valores plasmáticos preoperatorios de catepsina B no son significativamente más elevados en relación a un estadio tumoral más avanzado $(\mathrm{p}=0,549)$. Los valores inmunohistoquímicos de catepsina B tampoco fueron significativamente más elevados en relación a un estadio anatomopatológico tumoral más avanzado, $\mathrm{p}=0,823$.

Existió ralción significativa entre los valores inmunohistoquímicos de p53 y los valores determinados de catepsina B, en suero y tras estudio inmunohistoquímico, $\mathrm{p}=0,000$ y $\mathrm{p}=0,001$ respectivamente.

Las determinaciones inmunohistoquímicas de p53 y catepsina B no se relacionaron con la recidiva de la enfermedad, $\mathrm{p}=0,078$ y $\mathrm{p}=0,759$ respectivamente. La determinación sérica 


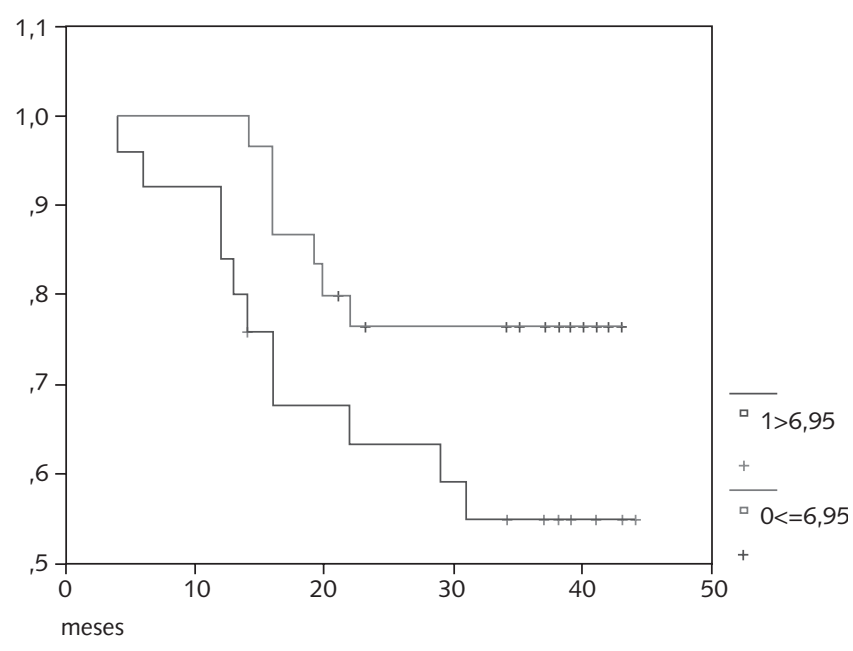

Fig. 2. Curvas de supervivencia de Kaplan-Meier en relación a valores dicotómicos de catepsina $B$ preoperatorios considerando la media como valor de corte.

de catepsina B preoperatoria no se relacionó con recidiva de la enfermedad, $\mathrm{p}=0,102$. Sólo los niveles de CEA preoperatorios se relacionaron con la recidiva neoplásica, $\mathrm{p}=0,005 ; \mathrm{HR}$ $1,005,95 \%$ IC (1,001-1,009).

La determinación preoperatoria se catepsina $\mathrm{B}$ se relacionó con una menor supervivencia del enfermo, $\mathrm{p}=0,041 ;$ HR $1,28195 \%$ IC $(1,010-1,624)$. Del mismo modo el resto de valores preoperatorios CEA y CA 19,9, se relacionaron con una menor supervivencia del enfermo, $\mathrm{p}=0,000$ y $\mathrm{p}=0,01$ respectivamente. No así la determinación de catepsina $B$ tisular, $p=0,13$. En un modelo multivariante que incluyó las determinaciones séricas preoperatorias, confirmó la relación de los valores de catepsina B de forma preoperatoria con la mortalidad del enfermo, $\mathrm{p}=0,022$, HR $1,338,95 \%$ IC $(1,043-1,716)$.

\section{DISCUSIÓN}

La presencia de metastatización locoregional y/o a distancia de la enfermedad colorrectal maligna constituye la principal causa de fallecimiento del enfermo. Aproximadamente un tercio de los enfermos con cáncer colorrectal sin metastatización ganglionar recidivan, debido al fracaso en la detección de enfermedad maligna oculta bien por la realización de una linfadenectomía insuficiente o por la existencia de micrometástasis no detectadas por los métodos histológicos habituales.

En nuestra hipótesis inicial consideramos que la expresión de ciertas enzimas implicadas en el proceso metastásico, como la Catepsina B, puede predecir el potencial metastásico y mala evolución de las neoplasias en etapas tempranas para considerar terapias más agresivas.

La invasión tumoral y metastatización neoplásica colorrectal a distancia precisa de la activación de varios sistemas proteolíticos que permitan a las células neoplásicas alcanzar el torrente circulatorio a través de la membrana basal y matriz extracelular.Los sistemas proteolíticos impli- cados en procesos antiadhesivos y migratorios en el proceso metastásico incluirá varias proteasas como son las metaloproteinasas, el activador del plasminogeno tipo-urokinasa (uPA) y las catepsinas, entre las cuales se incluirá la catepsina B.

La catepsina B es una cisteina proteinasa lisosómica que permite el progreso de la tumoración neoplásica degradando la membrana basal y matriz extracelular, directamente mediante su propia actividad lítica, o bien indirectamente mediante la degradación de inhibidores proteicos de otras proteasas, o activando proenzimas como pro-uPA (2-4).

Desde el inicio del estudio descartamos su utilidad como marcador diagnóstico tumoral específico del cáncer colorrectal dada su aparición en condiciones benignas (5), y existir referencias en la literatura médica que relacionan a la catepsina B con otras células neoplásicas no colorrectales (6-8). Sin embargo en nuestro estudio sí encontramos valores superiores de catepsina $\mathrm{B}$, tanto en determinación sérica como en estudio inmunohistoquímico en enfermos con cáncer colorrectal respecto a patología abdominal inflamatoria. Los valores de catepsina B preoperatorios en enfermos con cáncer colorrectal fueron significativamente superiores respecto al grupo control, $\mathrm{p}=0,011$, así como los valores de catepsina $\mathrm{B}$ inmunohistoquímicos, $\mathrm{p}=0,000$.

Tambien intentamos conocer su relación con los diferentes estadios anatomopatológicos,principal factor pronóstico hoy en día, y conocer su significación pronóstica. Las referencias bibliográficas son contradictorias respecto a los valores de catepsina $\mathrm{B}$ determinados en la pieza resecada. Autores como Adison y cols. (9), y Campo y cols. (10), consideran que los valores de catepsina B son más elevados en relación a la progresión neoplásica y por lo tanto la estadificación es más elevada. Sin embargo Murnane y cols. (11), consideran que los valores de catepsina B son superiores en etapas tempranas, estadios A y B de Dukes, en relación al enorme papel desempeñado por la catepsina B. Los valores de catepsina $\mathrm{B}$ inmunohistoquímicos no fueron significativamente superiores respecto a un estadio tumoral más avanzado, $\mathrm{p}=0,823$, quizás por la diversidad de enfermos en relación a los diferentes estadios anatomopatológicos. No obstante en nuestros enfermos los valores de catepsina B séricos preoperatorios sí se relacionaron significativamente con una menor supervivencia del enfermo, $\mathrm{p}=0,041 ;$ HR 1,281, 95\% IC (1,010-1,624). Su valor pronóstico independiente de las variables clinicopatológicas habitualmente utilizadas, se comparó con el de los marcadores tumorales pronóstico utilizados en cáncer colorrectal. En un modelo multivariante en el que incluimos determinaciones séricas preoperatorias de CEA, CA 19,9 y catepsina B, en relación a la mortalidad del enfermo, la catepsina $\mathrm{B}$ volvió a comportarse como un buen marcador pronóstico en relación a la supervivencia del enfermo, $\mathrm{p}=0,022$; HR 1,338, 95\% IC $(1,043-1,716)$.

Los resultados, a pesar de la escasa muestra, en relación a la determinación sérica preoperatoria de catepsina B y su utilización como marcador pronóstico en cáncer colorrectal, puede resultar esperanzador con la idea de identificar de aquellas neoplasias colorrectales de mala evolución.

Además existen nuevas alternativas terapeuticas basadas en el conocimiento profundo del proceso metastásico del cáncer colorrectal y el papel desempeñado por la catepsina B en dicho proceso (12). 


\section{Bibliografía}

1. Midis GP, Feig BW. Cancer of the Colon, Rectum and Anus. En: Feig BW, Berger DH, Fuhrman GM editors. The M.D. Anderson Surgical Oncology Handbook, 2nd ed. Philadelphia: Lippincott Wiliams \& Wilkins; 1999. p. 178-222.

2. Herszényi L, Plebani M, Carraro P, De Paoli M, Roveroni G, Cardin M, et al. The role of cysteine and serine proteases in colorectal carcinoma.Cancer 1999; 86: 1135-1142.

3. Yan S, Sameni M, Sloane BF.Cathepsin B and human tumor progression. Biol Chem 1998; 379: 113-23.

4. Mort JS, Buttle DJ.Cathepsin B. Int J Biochem Cell Biol 1997; 29: 715-720.

5. Jochum M, Billing AG, Frohlich D, Schildberg FW, Machleidt W, Cheronis JC et al. Proteolytic destruction of functional proteins by phagocytes in human peritonitis.Eur J Clin Invest 1999; 29: 246-55.

6. Watanabe M, Higashi T, Hashimoto M, Tomoda I, Tominaga S, Hashimoto M, et al. Elevation of tissue cathepsin B and L activities in gastric cancer. Hepato Gastroenterol 1987; 4:120-122.

7. Krepela E, Vicar J, Cernoch V. Cathepsin B in human breast tumor tissue and cancer cells. Neoplasma 1989; 36: 41-52.
8. Frohlich E, Schaumburg-Lever S, Klessen C.Inmunocytochemical and inmunoelectron microscopic demonstration of cathepsin B in human malignant melanoma. Br J Dermatol 1995; 132: 867-875.

9. Adenis A, Huet G, Zerimech F, Hecquet B, Balduyck M, Peyrat JP Cathepsin B, L, and D activities in colorectal carcinomas: relationship with clinicopathological parameters. Cancer Letters 1995; 96: 267-275.

10. Campo E, Muñoz J, Miquel R, Palacín A, Cardesa A, Sloane BF, et al. Cathepsin B expression in colorectal carcinomas correlates with tumour progression and shortened patient survival. Am J Pathol 1994; 145 (2): 301-309.

11. Murnane MJ, Sheahan K, Ozdemirli M, Shuja S. Stage-specific increases in Cathepsin B messenger RNA content in human colorectal carcinoma.Cancer Res 1991, 51: 1137-1142.

12. Muehlenwerg B, Assfalg-Machleidt I, Gil Parrado S, Bürgle M, Creutzburg S, Schmitt M, et al. A novel type of bifunctional inhibitor directed against proteolytic activity and receptor/ligand interaction. J Biol Chem 2000; 43: 33562-33566. 